\title{
The Singularity May Never Be Near
}

\author{
Toby Walsh
}

The technological singularity, often simply called the singularity, is the hypothesis that at some point in the future we will invent machines that can recursively self improve, and that this will be a tipping point resulting in runaway technological growth. I examine half a dozen arguments against this idea. 


\section{The History of the Singularity}

The idea of a technological singularity can be traced back to a number of different thinkers. Following John von Neumann's death in 1957, Stanislaw Ulam wrote:

One conversation [with John von Neumann] centered on the ever accelerating progress of technology and changes in the mode of human life, which gives the appearance of approaching some essential singularity in the history of the race beyond which human affairs, as we know them, could not continue. (Ulam 1958)

I. J. Good made a more specific prediction in 1965, calling it an "intelligence explosion" rather than a "singularity":

Let an ultraintelligent machine be defined as a machine that can far surpass all the intellectual activities of any man however clever. Since the design of machines is one of these intellectual activities, an ultraintelligent machine could design even better machines; there would then unquestionably be an intelligence explosion, and the intelligence of man would be left far behind. Thus the first ultraintelligent machine is the last invention that man need ever make." (Good 1965)

Many credit the technological singularity to the computer scientist, and science fiction author, Vernor Vinge, who predicted:

Within thirty years, we will have the technological means to create superhuman intelligence. Shortly after, the human era will be ended. (Vinge 1993)

More recently, the idea of a technological singularity has been popularized by Ray Kurzweil (2006) as well as others. Based on current trends, Kurzweil predicts the technological singularity will happen around 2045. For the purposes of this article, I suppose that the technological singularity is the point in time at which we build a machine of sufficient intelligence that is able to redesign itself to improve its intelligence, and at which its intelligence starts to grow exponentially fast, quickly exceeding human intelligence by orders of magnitude.

I start with two mathematical quibbles. The first quibble is that the technological singularity is not a mathematical singularity. The function $1 / 1-t$ has a mathematical singularity at $t=1$. This function demonstrates hyperbolic growth. As $t$ approaches 1 , its derivative ceases to be finite and well defined. Many proponents of a technological singularity argue only for exponential growth. For exampe, the function $2^{t}$ demonstrates exponential growth. Such an exponential function approaches infinity more slowly, and has a finite derivative that is always well defined. The second quibble is that the idea of exponential growth in intelligence depends entirely on the scale used to measure intelligence. If we measure intelligence in logspace, exponential growth is merely linear. I will not tackle here head on what we mean by measuring the intelligence of machines (or of humans). I will simply suppose there is such a prop- erty as intelligence, that it can be measured and compared, and that the technological singularity is when this measure increases exponentially fast in an appropriate and reasonable scale.

The possibility of a technological singularity has driven several commentators to issue dire predictions about the possible impact of artificial intelligence on the human race. For instance, in December 2014, Stephen Hawking told the BBC:

The development of full artificial intelligence could spell the end of the human race.... It would take off on its own, and re-design itself at an ever increasing rate. Humans, who are limited by slow biological evolution, couldn't compete, and would be superseded. ${ }^{1}$

Several other well known figures including Bill Gates, Elon Musk, and Steve Wozniak have subsequently issued similar warnings. Nick Bostrom has predicted a technological singularity, and argued that this poses an existential threat to the human race (Bostrom 2014). In this article, I will explore arguments as to why a technological singularity might not be close.

\section{Some Arguments Against the Singularity}

The idea of a technological singularity has received more debate outside the mainstream AI community than within it. In part, this may be because many of the proponents for such an event have come from outside this community. The technological singularity also has become associated with some somewhat challenging ideas like life extension and transhumanism. This is unfortunate as it has distracted debate from a fundamental and important issue: will we able to develop machines that at some point are able to improve their intelligence exponentially fast and that quickly far exceed our own human intelligence? This might not seem a particularly wild idea. The field of computing has profited considerably from exponential trends. Moore's law has predicted with reasonable accuracy that the number of transistors on an integrated circuit (and hence the amount of memory in a chip) will double every two years since 1975. And Koomey's law has accurately predicted that the number of computations per joule of energy dissipated will double every 19 months since the 1950s. Is it unreasonable to suppose AI will also at some point witness exponential growth?

The thesis put forward here is that there are several strong arguments against the possibility of a technological singularity. Let me be precise. I am not predicting that AI will fail to achieve superhuman intelligence. Like many of my colleagues working in AI, I predict we are just 30 or 40 years away from this event. However, I am suggesting that there will not be the runaway exponential growth predicted by some. I will put forward multiple arguments why a technological singularity is improbable.

These are not the only arguments against a tech- 
nological singularity. We can, for instance, also inherit all the arguments raised against artificial intelligence itself. Hence, there are also the nine common objections considered by Alan Turing in his seminal Mind paper (Turing 1963) like machines not being conscious, or not being creative. I focus here though on arguments that go to the idea of an exponential runaway in intelligence.

\section{The Fast Thinking Dog Argument}

One of the arguments put forwards by proponents of the technological singularity is that silicon has a significiant speed advantage over our brain's wetware, and this advantage doubles every two years or so according to Moore's law. Unfortunately speed alone does not bring increased intelligence. To adapt an idea from Vernor Vinge (1993), a faster thinking dog is still unlikely to play chess. Steven Pinker put this argument eloquently:

There is not the slightest reason to believe in a coming singularity. The fact that you can visualize a future in your imagination is not evidence that it is likely or even possible. Look at domed cities, jet-pack commuting, underwater cities, mile-high buildings, and nuclear-powered automobiles - all staples of futuristic fantasies when I was a child that have never arrived. Sheer processing power is not a pixie dust that magically solves all your problems. (Pinker 2008)

Intelligence is much more than thinking faster or longer about a problem than someone else. Of course, Moore's law has certainly helped AI. We now learn off bigger data sets. We now learn quicker. Faster computers will certainly help us build artificial intelligence. But, at least for humans, intelligence depends on many other things including many years of experience and training. It is not at all clear that we can short circuit this in silicon simply by increasing the clock speed.

\section{The Anthropocentric Argument}

Many descriptions of the technological singularity supposes human intelligence is some special point to pass, some sort of "tipping" point. For instance, Nick Bostrom writes:

"Human-level artificial intelligence leads quickly to greater-than-human-level artificial intelligence. . . . The interval during which the machines and humans are roughly matched will likely be brief. Shortly thereafter, humans will be unable to compete intellectually with artificial minds." (Bostrom 2002)

Human intelligence is one point on a wide spectrum that takes us from cockroach through mouse to human. Actually, it might be better to say it is a probability distribution rather than a single point. It is not clear in arguments like the above which level of human intelligence requires to be exceeded before runaway growth kicks in. Is it some sort of average intelligence? Or the intelligence of the smartest human ever?

If there is one thing that we should have learned from the history of science, it is that we are not as special as we would like to believe. Copernicus taught us that the universe did not revolve around the earth. Darwin taught us that we were little different from the apes. And artificial intelligence will likely teach us that human intelligence is itself nothing special. There is no reason therefore to suppose that human intelligence is some special tipping point that, once passed, allows for rapid increases in intelligence. Of course, this doesn't preclude there being some level of intelligence that is a tipping point.

One argument put forwards by proponents of a technological singularity is that human intelligence is indeed a special point to pass because we are unique in being able to build artefacts that amplify our intellectual abilities. We are the only creatures on the planet with sufficient intelligence to design new intelligence, and this new intelligence will not be limited by the slow process of reproduction and evolution. However, this sort of argument supposes its conclusion. It assumes that human intelligence is enough to design an artificial intelligence that is sufficiently intelligent to be the starting point for a technological singularity. In other words, it assumes we have enough intelligence to initiate the technological singularity, the very conclusion we are trying to draw. We may or may not have enough intelligence to be able to design such artificial intelligence. It is far from inevitable. Even if we have enough intelligence to design superhuman artificial intelligence, this superhuman artificial intelligence may not be adequate to percipitate a technological singularity.

\section{The Metaintelligence Argument}

One of the strongest arguments against the idea of a technological singularity in my view is that it confuses intelligence to do a task with the capability to improve your intelligence to do a task. David Chalmers, in an otherwise careful analysis of the idea of a technological singularity, writes: "If we produce an AI by machine learning, it is likely that soon after we will be able to improve the learning algorithm and extend the learning process, leading to AI+" (Chalmers 2010).

Here, AI is a system with human-level intelligence and $\mathrm{AI}+$ is a system more intelligent than the most intelligent human. But why should it be likely that soon after we can improve the learning algorithm? Progress in machine-learning algorithms has neither been especially rapid or easy. Machine learning is indeed likely to be a significant component of any human-level AI system that we might build in the future if only because it will be painful to hand code its knowledge and expertise otherwise. Suppose an AI system uses machine learning to improve its performance at some tasks requiring intelligence like understanding a text, or proving mathematical identities. There is no reason that the system can in addition improve the fundamental machine-learning algo- 
rithm used to do this. Machine-learning algorithms frequently top out a particular task, and no amount of tweaking, be it feature engineering or parameter tuning, appears able to improve their performance.

We are currently seeing impressive advances in AI using deep learning (Edwards 2015). This has dramatically improved the state of the art in speech recognition, computer vision, natural language processing, and a number of other domains. These improvements have come largely from using larger data sets, and deeper neural networks:

Before, neural networks were not breaking records for recognizing continuous speech; they were not big enough. (Yann LeCun, quoted in Edwards [2015]).

Of course, more data and bigger neural networks means we need more processing power. As a result, GPUs are now frequently used to provide this processing power. However, being better able to recognize speech or identify objects has not led to an improvement in deep learning itself. The deep-learning algorithms have not improved themselves. Any improvements to the deep-learning algorithms have been hard won by applying our own intelligence to their design.

We can come at this argument from another direction using one of the best examples we know of intelligent systems. Look at ourselves. It is much easier for us to learn how to do better at a particular task than it is for us to learn how to learn better in general. For instance, if we remove the normalization inherent in the definition of IQ, we can observe that IQ has increased over the last century but only slowly (the "Flynn effect"). And improving your IQ today is pretty much as slow and painful as it was a century ago. Perhaps electronic brains will also struggle to improve their performance quickly and never get beyond a fraction of their fundamental capabilities?

\section{The Diminishing Returns Argument}

The idea of a technological singularity typically supposes improvements to intelligence will be a relative constant multiplier, each generation getting some fraction better that the last. However, the performance so far of most of our AI systems has been that of diminishing returns. There is often lots of low-hanging fruit at the start, but we then run into great difficulties to improve after this. This helps explain the overly optimistic claims made by many of the early AI researchers. An AI system may be able to improve itself an infinite number of times, but the extent to which its intelligence changes overall could be bounded. For instance, if each generation only improves by half the last change, then the system will never get beyond doubling its overall intelligence.

Diminishing returns may also come not from the difficulty of improving our AI algorithms, but from the difficulty of their subject matter increasing rapidly. Paul Allen, the Microsoft cofounder, calls this the "complexity brake."
We call this issue the complexity brake. As we go deeper and deeper in our understanding of natural systems, we typically find that we require more and more specialized knowledge to characterize them, and we are forced to continuously expand our scientific theories in more and more complex ways ... we believe that progress toward this understanding [of cognition] is fundamentally slowed by the complexity brake. (Allen and Greaves 2011)

Even if we see continual, perhaps even exponential improvements in our AI systems, this may not be enough to improve performance. The difficulty of the problems required to be solved to see intelligence increase may themselves increase even more rapidly. There are those that argue theoretical physics appears to be running into such complexity brakes.

\section{The Limits of Intelligence Argument}

There are many fundamental limits within the universe. Some of these are physical. You cannot accelerate past the speed of light. You cannot know both position and momentum with complete accuracy. You cannot know when the radioactive decay of an atom will happen with certainty. Any thinking machine that we might build will be limited by these physical laws. Of course, if that machine is electronic or even quantum in nature, these limits are likely to be much greater than the biological and chemical limits of our human brains.

There are also more empirical laws that can be observed emerging out of complex systems. For example, Dunbar's number is the observed correlation between brain size for primates and average social group size. This puts a limit of between 100 and 250 stable relationships on human social groups. Intelligence is also a complex phenomenon and may also have such limits that emerge from this complexity. Any improvements in machine intelligence, whether it runs away or happens more slowly, may run into such limits. Or course, there is no reason to suppose that our own human intelligence is at or close to this limit. But equally, there's little reason why any such limits are necessarily far beyond our own intelligence.

\section{The Computational Complexity Argument}

Suppose we stick to building AI systems with computers that obey our traditional models of computation. Even exponential improvements are no match for computational complexity. For instance, exponential growth in performance is inadequate to run superexponential algorithms. And no amount of growth in performance will make undecidable problems decidable. Computational complexity may be one of the fundamental limits discussed in the previous argument. Hence, unless we use machines that go beyond our traditional models of computation, we are likely to bump into many problems where computational complexity fundamentally limits performance. Of course, a lot of computational complexity is 


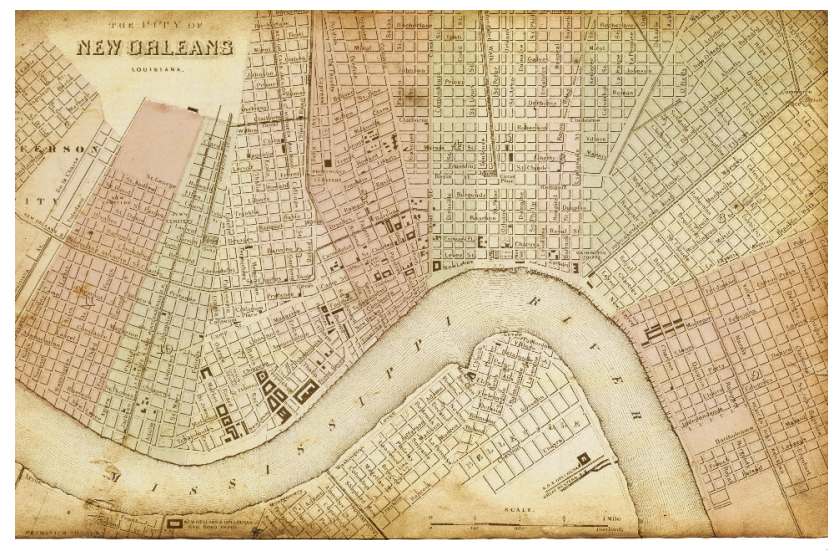

Courtesy, iStock

Join Us in New Orleans for AAAI-18!

AAAI-18 will arrive in New Orleans just prior to Mardi Gras and festivities will already be underway. Enjoy legendary jazz music, the French Quarter filled with lively clubs and restaurants, world-class museums, and signature architecture. New Orleans' multicultural and diverse communities will make your experience in the Big Easy unique. The Hilton New Orleans Riverside is located in the thriving Warehouse and Arts District and is an easy walk to the French Quarter, the Audubon Aquarium, and the Butterfly Garden and Zoo. For complete information about options in New Orleans, please see visit www.neworleanscvb.com/visit.

\section{Please join us in 2017 in New Orleans for a memorable AAAI!}

\section{www.aaai.org/aaai18}

about worst case, and much of $\mathrm{AI}$ is about using heuristics to solve problems in practice that are computationally intractable in the worst case. There are, however, fundamental limits to the quality of these heuristics. There will be classes of problems that even a superhuman intelligence cannot solve well, even approximately.

\section{Conclusions}

I have argued that there are many reasons why we might never witness a technological signularity. Nevertheless, even without a technological singularity, we might still end up with machines that exhibit super- human levels of intelligence. We might just have to program much of this painfully ourselves. If this is the case, the impact of AI on our economy, and on our society, may be less dramatic than either the pessimists or the optimists have predicted. Nevertheless, we should start planning for the impact that AI will have on society. Even without a technological singularity, AI is likely to have a large impact on the nature of work. As a second example, even quite limited AI is likely to have a large impact on the nature of war. We need to start planning today for this future.

\section{Acknowledgements}

The author is supported by the European Research Council and the Asian Office of Aerospace Research and Development.

\section{Note}

1. See Rory Cellan-Jones, Stephen Hawking Warns Artificial Intelligence Could End Mankind, BBC News, Technology, 2 December 2014. (www.bbc.co.uk/news/technology30290540).

\section{References}

Allen, P., and Greaves, M. 2011. The Singularity Isn't Near. MIT Technology Review (March/April), 7-65.

Bostrom, N. 2002. When Machines Outsmart Humans. Futures 35(7): 759-764. doi.org/10.1016/S0016-3287(03) 00026-0

Bostrom, N. 2014. Superintelligence: Paths, Dangers, Strategies. Oxford, UK: Oxford University Press.

Chalmers, D. 2010. The Singularity: A Philosophical Analysis. Journal of Consciousness Studies 17(9-10): 7-65.

Edwards, C. 2015. Growing Pains for Deep Learning. Communications of the ACM 58(7): 14-16. doi.org/10.1145/ 2771283

Good, I. 1965. Speculations Concerning the First Ultraintelligent Machine. Advances in Computers 6: 31-88. Amsterdam, The Netherlands: Elsevier. doi.org/10.1016/S00652458(08)60418-0

Kurzweil, R. 2006. The Singularity Is Near: When Humans Transcend Biology. London: Penguin.

Pinker, S. 2008. Tech Luminaries Address Singularity. IEEE Spectrum. Special Report: The Singularity, June 1.

Turing, A. 1963. Computing Machinery and Intelligence. In Computers and Thought. New York: McGraw-Hill Book Co.

Ulam, S. 1958. Tribute to John von Neumann. Bulletin of the American Mathematical Society 64(3).

Vinge, V. 1993. The Coming Technological Singularity: How to Survive in the Post-Human Era. In Whole Earth Review, ed. H. Rheingold. San Francisco: Point Foundation.

Toby Walsh is a guest professor at TU Berlin, the Scientia Professor of Artificial Intelligence at the University of New South Wales, and leads the Algorithmic Decision Theory group at Data61, Australia's Centre of Excellence for ICT Research. He has been elected a Fellow of the Australian Academy of Science and of the Association for the Advancement of Artificial Intelligence. He has won the Humboldt research award and the NSW Premier's Prize for Excellence in Engineering and ICT. 